\title{
Salusin- $\alpha$ Inhibits Proliferation and Migration of Vascular Smooth Muscle Cell via Akt/mTOR Signaling
}

\author{
Shoucui Gao ${ }^{a, b} \quad$ Liran Xu $u^{a, b} \quad$ Yali Zhang ${ }^{a, b} \quad$ Qingqing Yua,b Jiayan Li ${ }^{a}$ \\ Hua Guan ${ }^{a, b} \quad$ Xiaojing Wanga,b Daxin Chenga,b Yi Liu,a,b Liang Baia,b \\ Rong Wang ${ }^{a, b}$ Jianglin Fanc Sihai Zhao ${ }^{a, b, d}$ Enqi Liu
}

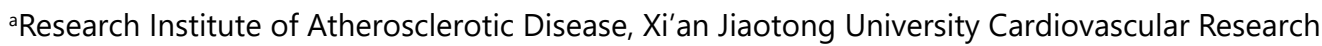
Center, Xi'an, 'Laboratory Animal Center, Xi'an Jiaotong University Health Science Center, Xi'an, China, 'Department of Molecular Pathology, Interdisciplinary Graduate School of Medicine, University of Yamanashi, Yamanashi, Japan, dDepartment of Vascular Surgery, Stanford University School of Medicine, Stanford, USA

\section{Key Words}

Salusin- $\alpha \cdot$ Smooth muscle cell $\bullet$ Proliferation $\cdot$ Atherosclerosis

\begin{abstract}
Background/Aims: The proliferation and migration of vascular smooth muscle cells (VSMCs) are key steps in the progression of atherosclerosis. The aim of the present study was to investigate the potential roles of salusin- $\alpha$ in the functions of VSMCs during the development of atherosclerosis. Methods: In vivo, the effects of salusin- $\alpha$ on atherogenesis were examined in rabbits fed a cholesterol diet. The aortas were en face stained with Sudan IV to evaluate the gross atherosclerotic lesion size. The cellular components of atherosclerotic plaques were analyzed by immunohistochemical methods. In vitro, Cell Counting Kit- 8 and wound-healing assays were used to assess the effects of salusin- $\alpha$ on VSMC proliferation and migration. In addition, western blotting was used to evaluate the total and phosphorylated levels of Akt (also known as protein kinase B) and mammalian target of rapamycin (mTOR) in VSMCs. Results: Salusin- $\alpha$ infusion significantly reduced the aortic lesion areas of atherosclerosis, with a $39 \%$ reduction in the aortic arch, a $71 \%$ reduction in the thoracic aorta, and a $71 \%$ reduction in the abdominal aorta; plasma lipid levels were unaffected. Immunohistochemical staining showed that salusin- $\alpha$ decreased both macrophage- and VSMC-positively stained areas in atherosclerotic lesions by $54 \%$ and $69 \%$, cell proliferative activity in the intima and media of arteriosclerotic lesions, and matrix metalloproteinase 2 (MMP-2) and MMP-9 expression in plaques. Studies using cultured VSMCs showed that salusin- $\alpha$ decreased VSMC migration and proliferation via reduced phosphorylation of Akt and mTOR. Conclusion: Our data indicate that salusin- $\alpha$ suppresses the development of atherosclerosis by inhibiting VSMC proliferation and migration through the Akt/mTOR pathway.




\section{Cellular Physiology Cell Physiol Biochem 2018;50:1740-1753

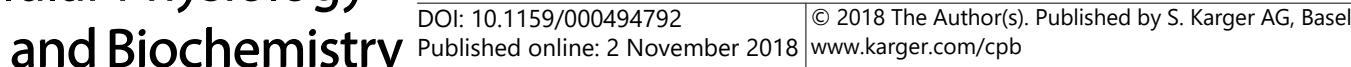

Gao et al.: Salusin- $\alpha$ Inhibits Vascular Smooth Muscle Cell Proliferation

\section{Introduction}

Atherosclerosis is a chronic disease characterized by the accumulation of lipid, inflammatory cells and the proliferation of arterial smooth muscle cells (SMCs) [1-3]. The pathogenesis of atherosclerosis is a multifactorial process involving several molecular pathways. It may be modulated by endogenous vasoconstrictor peptides, such as adropin, angiotensin II, endothelin-1, vaspin, and urotensin II [4-8]. These agents stimulate vascular inflammation and oxidative stress-induced endothelial injury, monocyte-endothelial cell adhesion, macrophage foam cell formation, and SMC migration and proliferation, which are the pivotal phenomena of atherosclerosis [9]. Of all of the candidate biomarkers extensively studied so far, the recently identified bioactivities of salusins in atherogenesis are of special note. Salusin- $\alpha$ and $-\beta$, endogenous peptides biosynthesized from the same precursor, prosalusin, exert diverse effects on the cardiovascular system. The salusins are translated from an alternatively spliced mRNA of torsin family 2, member A. Salusin- $\alpha$ consists of 28 amino acids, whereas salusin- $\beta$ consists of 20 amino acids, and both are bioactive peptides with hemodynamic effects [10-12]. Salusins are expressed and synthesized ubiquitously within human and animal tissues and detected in both plasma and urine, suggesting that salusins are endocrine and/or paracrine factors [10,13,14].

Several lines of evidence suggest that salusins may be involved in the regulation of vascular homeostasis and play important roles in the development of cardiovascular disease. Atherosclerosis development is accelerated by salusin- $\beta$ via increased acyl-coenzyme A:cholesterol acyltransferase-1 expression in human monocyte-derived macrophages, whereas salusin- $\alpha$ may have the opposite effect: serum salusin- $\alpha$ levels are inversely correlated with the maximum intima-media thickness in patients with coronary artery disease $[15,16]$. Although there is a link between salusin- $\alpha$ and atherosclerosis $[11,16$, 17], it is unclear whether salusin- $\alpha$ plays a role in the proliferation and migration of SMCs, another important cellular event in atherosclerotic lesion formation, even though some reports have shown that salusin- $\beta$ promotes SMC migration and proliferation and vascular inflammation and fibrosis [18-20]. In this study, we attempted to examine the effects of salusin- $\alpha$ on rabbit atherosclerosis, with emphasis on the role of the peptide in SMCs and the underlying mechanisms.

\section{Materials and Methods}

\section{Animals}

Male Japanese white rabbits were supplied by the Laboratory Animal Center of Xi'an Jiaotong University. To avoid hemodynamic effects induced by intravenous bolus injection, we performed subcutaneous continuous infusion of human synthetic salusin- $\alpha$ peptide $(0.6 \mathrm{nmol} / \mathrm{kg} / \mathrm{h} ; 54847$, GL Biochem, Shanghai, China) with the use of osmotic mini-pumps (Alzet Model 2006; Durect, Cupertino, CA). The mini-pumps were loaded with either salusin- $\alpha$ dissolved in saline (vehicle) or vehicle only and placed in 4-month-old rabbits. Pumps were subcutaneously implanted into the back of the neck of rabbits anesthetized with isoflurane and replaced with new pumps every 4 weeks. Both groups of rabbits were fed a chow diet containing $0.3 \%$ cholesterol and 3\% soybean oil for 16 weeks. All animal experiments were performed with the approval of the Animal Care Committee of Xi'an Jiaotong University and conformed to the Guide for the Care and Use of Laboratory Animals published by the US National Institutes of Health.

\section{Measurement of body weight and plasma parameters}

Body weight and plasma lipids were recorded every 2 weeks in each group of rabbits. Blood samples were collected via the auricular artery using an EDTA anticoagulant tube after $16 \mathrm{~h}$ of food deprivation and then centrifuged (3000 rpm, $15 \mathrm{~min}, 4^{\circ} \mathrm{C}$ ) to obtain plasma. Plasma levels of total cholesterol (TC), triglycerides (TGs), and high-density lipoprotein-cholesterol (HDL-C) were measured using Biosino assay kits (Bio-technology \& Science Inc., Beijing, China). Plasma levels of tumor necrosis factor- $\alpha$ (TNF- $\alpha$ ) 


\section{Cellular Physiology Cell Physiol Biochem 2018;50:1740-1753

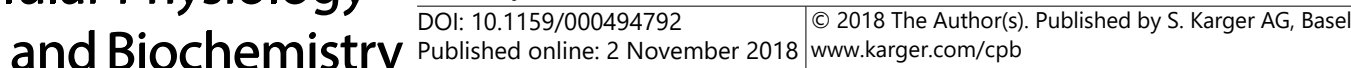

and interleukin-6 (IL-6) were measured with enzyme-linked immunosorbent assay kits according to the manufacturers' instructions (R\&D Systems Inc., Minneapolis, MN).

\section{Atherosclerotic lesion analysis}

At the end of the experiment, the rabbits were sacrificed via an overdose of sodium pentobarbital for analysis of atherosclerotic lesions. The entire aortic tree was fixed in 10\% neutral buffered formalin and stained with Sudan IV (Yongsheng Chemical Co., Ji'nan, China) as previously described [21]. The area of the atherosclerotic lesion (sudanophilic area) was measured using image analysis software (WinROOF 6.5, Mitani Co. Ltd., Tokyo, Japan). For microscopic quantification of the lesion area, each segment of the rabbit aorta was cut into cross sections (8 to 10 for the aortic arch as described previously) [22]. For histological analysis, the sections were embedded in paraffin and stained with hematoxylin and eosin, elastic van Gieson, and Masson's trichrome staining. For the microscopic evaluation of the cellular components in the lesions, serial paraffin sections of the aortic arch were immunohistochemically stained with antibodies (Abs) against macrophages (MФ) (1:500; RAM11, Dako Co., Carpinteria, CA) and SMCs (1:400; $\alpha$-actin; Dako Co.). To further explore the plaque components, we also performed Masson's trichrome and immunohistochemical staining with Abs against proliferating cell nuclear antigen (PCNA), matrix metalloproteinase 2 (MMP-2) (1:800; MAB3308, Millipore, Billerica, MA), and MMP-9 (1:50; IM37, Millipore). The sections for microscopic quantification were examined and photographed under a microscope equipped with a digital camera (Nikon, Tokyo, Japan) and measured with image analysis software.

\section{Cell culture}

We obtained VSMCs from the media of rat aortas by the tissue explant method [23] and cultured them in Dulbecco's modified Eagle's medium (DMEM) supplemented with 10\% fetal bovine serum (FBS), penicillin $(100 \mathrm{U} / \mathrm{mL})$, and streptomycin $(100 \mathrm{U} / \mathrm{mL})$. Briefly, male Sprague-Dawley rats $(\sim 250 \mathrm{~g})$ were humanely euthanized via pentobarbital overdose. The thoracic aortas were removed and turned "inside out" in FBS with the use of a unique catheter under a microscope. The aortas were then cut into 1-2-mm explants and the explants were individually plated in culture plates containing DMEM with 20\% FBS for 7 days until a single-cell suspension was achieved. The obtained cells retained the characteristics of SMCs (purity $>90 \%$ ). Cells from passages 3-9 at 80\% confluence in culture wells were used after serum depletion $24 \mathrm{~h}$ prior to treatment.

\section{Cell proliferation assay}

Cell Counting Kit-8 (CCK-8) kits (Dojindo, Tokyo, Japan) were used to measure VSMC proliferation according to the manufacturer's instructions. VSMCs were seeded in 96-well plates at a density of $4 \times 10^{3}$ cells/well and then divided into three treatment groups: a vehicle-treated group, platelet-derived growth factor-BB (PDGF-BB)-treated group (10 ng/mL), and PDGF-BB (10 ng/mL) + salusin- $\alpha$ (10 nM)-treated group. To further examine the role of mTOR in VSMC proliferation, MHY-1485 was applied to activate the mTOR signaling pathway. VSMCs were pretreated with PDGF-BB for $2 \mathrm{~h}$ and then divided into four treatment groups: a vehicle-treated group, MHY1485-treated group (10 $\mu \mathrm{M}$; S7811, Selleck, Houston, Texas), salusin- $\alpha$ $(10 \mathrm{nM})$-treated group, and MHY1485 $(10 \mu \mathrm{M})+$ salusin- $\alpha(10 \mathrm{nM})$-treated group. After incubation for $36 \mathrm{~h}$, $10 \mu \mathrm{l}$ of CCK-8 solution was added to each well and the cells were incubated for $2 \mathrm{~h}$ at $37^{\circ} \mathrm{C}$. The absorbance was measured at $450 \mathrm{~nm}$ with an Infinite 200 Pro plate reader (Tecan, Männedorf, Switzerland).

For a 5-ethynyl-2'-deoxyuridine (EdU) incorporation assay, the staining procedure was performed using a Cell-Light ${ }^{\mathrm{TM}}$ EdU Apollo® 488 In vitro Imaging Kit (RiboBio, Guangzhou, China) in accordance with the manufacturer's instructions. Cells were seeded onto 96 -well plates at a density of $4 \times 10^{3}$ cells/well and then divided into three treatment groups: a vehicle-treated group, PDGF-BB-treated group (10 ng/ $\mathrm{mL})$, and PDGF-BB (10 ng/mL) + salusin- $\alpha(10 \mathrm{nM})$-treated group. To examine the role of mTOR in VSMC proliferation, VSMCs were treated with PDGF-BB for $2 \mathrm{~h}$ and then divided into four groups: a vehicletreated group, MHY1485-treated group $(10 \mu \mathrm{M})$, salusin- $\alpha(10 \mathrm{nM})$-treated group, and MHY1485 (10 $\mu \mathrm{M})+$ salusin- $\alpha(10 \mathrm{nM})$-treated group. After incubation for $36 \mathrm{~h}$, EdU working solution was added to the cultures and, $2 \mathrm{~h}$ later, the cells were collected and fixed with $4 \%$ paraformaldehyde. The cells were stained by Apollo ${ }^{\circledR}$ staining solution and Hoechst 33342. EdU-positive cells were counted and normalized to the total number of Hoechst 33342-stained cells. The fluorescence signals were observed via fluorescence microscopy (ECLIPSE Ti, Nikon), and images were analyzed using NIS-Elements F software (Nikon). 


\section{Cellular Physiology Cell Physiol Biochem 2018;50:1740-1753 \begin{tabular}{l|l|l} 
and Biochemistry Published online: 2 November 2018 & $\begin{array}{l}\text { ○ } 2018 \text { The Author(s). Published by S. Karger AG, Basel } \\
\text { www.karger.com/cpb }\end{array}$ \\
\hline
\end{tabular}}

\section{VSMC migration assay}

VSMC migration was assessed by a wound-induced migration assay [23]. In brief, VSMCs were seeded in 6-well plates at $10^{5}$ cells/well and cultured in DMEM supplemented with $10 \%$ FBS and antibiotics until they reached $80 \%$ confluence. The quiescent VSMCs were then scraped in a straight line with a P1000 pipette tip to create a "scratch", and then the cells were washed twice with $1 \mathrm{~mL}$ of PBS. The medium was then replaced with $1 \mathrm{~mL}$ of medium specific for the in vitro scratch assay. The in vitro study procedure and chemical treatment were the same as in the above proliferation assay. The wound-healing images were captured in $40 \times$ fields at 0 and 24 h post-wounding under a Nikon microscope (Nikon) equipped with Image-pro Plus software (Media Cybernetics Inc., Bethesda, MD). The ratio of the cell recovery area to the whole wound area was used to evaluate cell migration.

\section{Western blot analysis}

VSMCs were pretreated with different concentrations (1 and $10 \mathrm{nM}$ ) of salusin- $\alpha$ before stimulation with PDGF-BB (10 ng/mL) for $24 \mathrm{~h}$. Briefly, the VSMC culture medium was removed and the cells were washed three times with PBS. VSMCs were then resuspended in lysis buffer: $50 \mathrm{mmol} / \mathrm{L}$ Tris- $\mathrm{HCl}, \mathrm{pH} \mathrm{6.8,}$ $15 \%$ glycerol, $5 \% \beta$-mercaptoethanol, $2 \%$ sodium dodecyl sulfate, $0.001 \%$ bromophenol, and protease inhibitor cocktail (Roche, Indianapolis, IN). The debris was removed and the supernatant was obtained by centrifugation at $12,000 \mathrm{~g}$ for $10 \mathrm{~min}$ at $4^{\circ} \mathrm{C}$. Protein concentrations were determined using the BCA protein assay kit (Pierce, Waltham, MA). Lysates containing $30 \mu \mathrm{g}$ of protein were analyzed by $10 \%$ sodium dodecyl sulfate-polyacrylamide gel electrophoresis and transferred onto a polyvinylidene fluoride membrane. The membrane was blocked with 5\% BSA in PBS and then probed with the desired primary Abs at $4{ }^{\circ} \mathrm{C}$ overnight. The blots were developed using appropriate horseradish peroxidase-conjugated secondary Abs (1:2000; 7074, Cell Signaling Technology, Beverly, MA) and an ECL Plus system (Thermo Fisher Scientific, Waltham, MA). The band intensity was analyzed by densitometry using Image software. GAPDH protein served as a loading control. Protein samples were western blotted against primary Abs for p-Akt $(1: 1000 ; 4060)$, t-Akt (1:1000; 4691), p-Erk1/2 (1:1000; 4370S), t-Erk1/2 (1:1000; 4695), p-p38 MAPK (1:1000; 4511), t-p38 MAPK (1:1000; 8690S), p-mTOR (1:1000; 5536), t-mTOR (1:1000; 2983) (all from Cell Signaling Technology), and GAPDH (1:2000; ab181602, Abcam, Cambridge, UK).

\section{Quantitative real-time polymerase chain reaction}

VSMCs were divided into three treatment groups: a vehicle-treated group, PDGF-BB-treated group $(10 \mathrm{ng} / \mathrm{mL})$, and PDGF-BB $(10 \mathrm{ng} / \mathrm{mL})+$ salusin- $\alpha(10 \mathrm{nM})$-treated group. Total RNA was extracted from cells using TRIzol reagent (Invitrogen, Carlsbad, CA) by the RNeasy method following the manufacturer's instructions. Quantitative real-time polymerase chain reaction (RT-PCR) was performed by using SYBR Green technology (Applied Biosystems, Foster City, CA) and a 7500 Real-time PCR machine (Applied Biosystems). Fold changes in gene expression were calculated using the $2^{-\triangle \Delta C T}$ method. The qRT-PCR primers used were as follows: MMP-2 forward primer: 5'-TCCCGAGATC TGCAAGCAAG-3', reverse primer: 5'-AGAATGTGGC CACCAGCAAG-3'; MMP-9 forward primer: 5'-AGCCGGGAAC GTATCTGGA-3', reverse primer: 5'-TGGAAACTCA CACGCCAGAAG-3'; GAPDH forward primer: 5'-GAACCCTAAG GCCAACCGTG-3', reverse primer: 5'-TGGCATAGAG GTCTTTACGG-3'. GAPDH was used as an internal control.

\section{Statistical analysis}

Data are expressed as the mean \pm standard deviation. Statistical analysis was performed using Student's $t$-test with an equal $F$ value or Welch's t-test when the $F$ value was not equal. Data from three groups were analyzed by one-way analysis of variance, followed by Tukey's multiple-comparison procedure. For unequal variances, data were evaluated by the Kruskal-Wallis test. $P<0.05$ was considered significant. 


\section{Results}

Body weight and plasma parameters

There was no clear difference in body weight under the same food consumption conditions between the vehicletreated and salusin- $\alpha$-treated groups, neither at the start nor at the end of the experimental period (Fig. 1A). At the end of the in vivo study, the plasma TC level was substantially increased after the high cholesterol diet feeding (Fig. 1B). However, there were no significant differences in plasma lipids (TC, $\mathrm{TG}$, or HDL-C) or inflammation targets (numbers of blood monocytes or neutrophils and plasma TNF- $\alpha$ and IL- 6 levels) between the two groups (Fig. 1B-G), indicating that chronic infusion of salusin- $\alpha$ had no significant effects on these parameters.

\section{Aortic lesion formation}

Analysis of the relative en face aortic lesion areas revealed that the total aortic atherosclerotic lesion areas of the salusin- $\alpha$-treated group were significantly reduced by $59 \%$ versus the vehicle-treated group (Fig. 2A), with a 39\% reduction in the aortic arch, a $71 \%$ reduction in the thoracic aorta, and a $71 \%$ reduction in the abdominal aorta (Fig. 2B). Histological examination along with immunohistochemical staining showed that the atherosclerotic lesions of both salusin- $\alpha$ and control groups were mainly composed of fatty streaks (Fig. 2C). Similarly, the microscopic lesion size of the aortic arch was decreased by $59 \%$ in the salusin- $\alpha$-treated group due to decreased numbers of both macrophages (54\% decrease) and SMCs (69\% decrease) compared with the vehicle-treated group (Fig. 2D). Furthermore, the relative expression of PCNA was significantly lower in the salusin- $\alpha$-treated group than in the vehicle-treated group (Fig. 2E and F). These results indicated that salusin- $\alpha$ may also inhibit atherosclerotic lesion progression via inhibition of VSMC proliferation and/or migration. To evaluate the collagen content, we stained the sections with Masson's trichrome staining. The collagen content was higher (27\% increase) in the salusin- $\alpha$-treated group than in the vehicle-treated group (Fig. $2 \mathrm{G}$ and H).

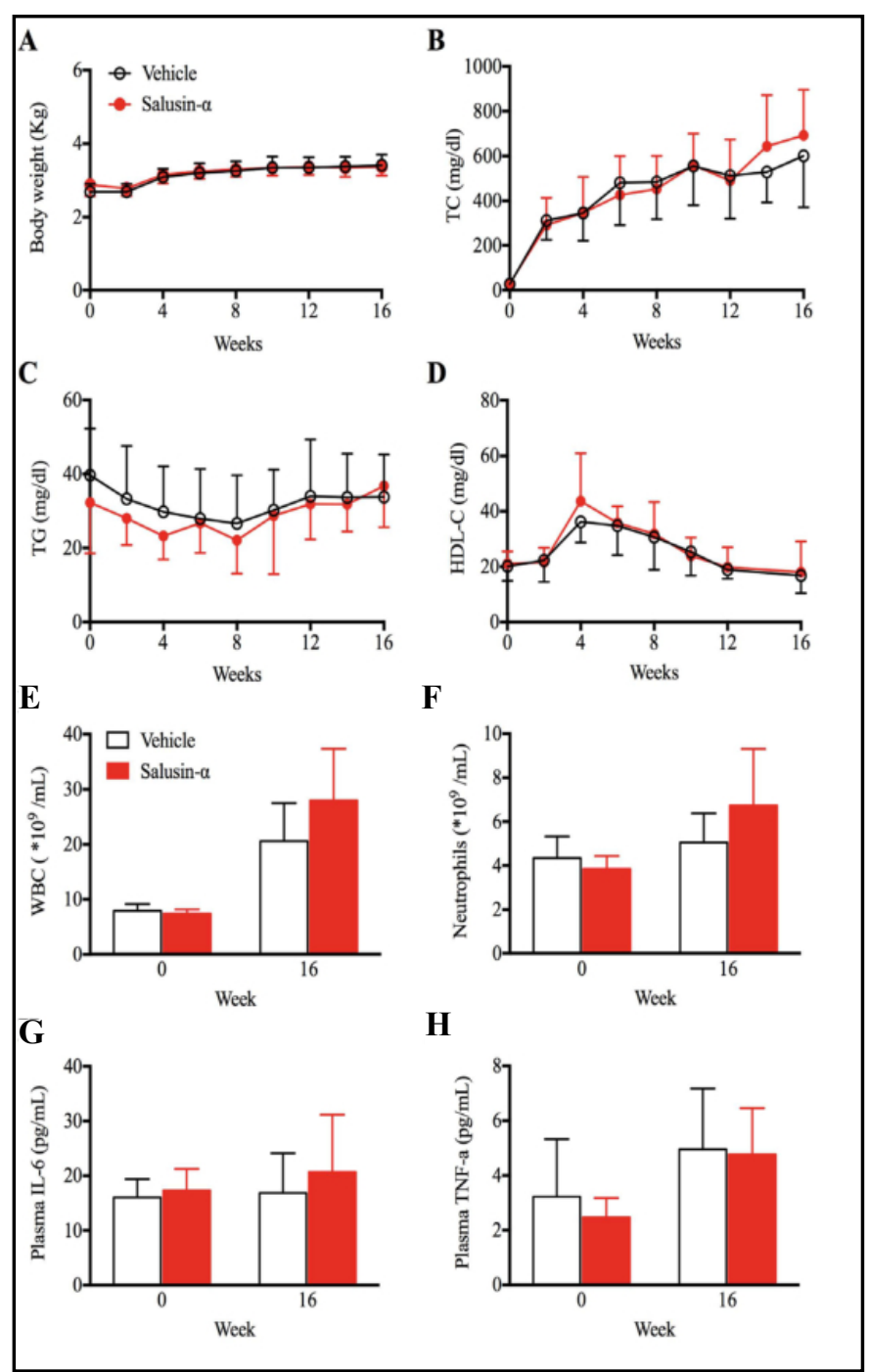

Fig. 1. Body weight, plasma lipid levels, and inflammatory profiles of rabbits fed a cholesterol-rich diet. BW, body weight; TC, total cholesterol; TG, triglyceride; HDL, high-density lipoprotein; WBC, white blood cell; TNF- $\alpha$, tumor necrosis factor- $\alpha$; and IL6 , interleukin-6. Data are expressed as the mean \pm standard deviation. $n=9$ for each group. 


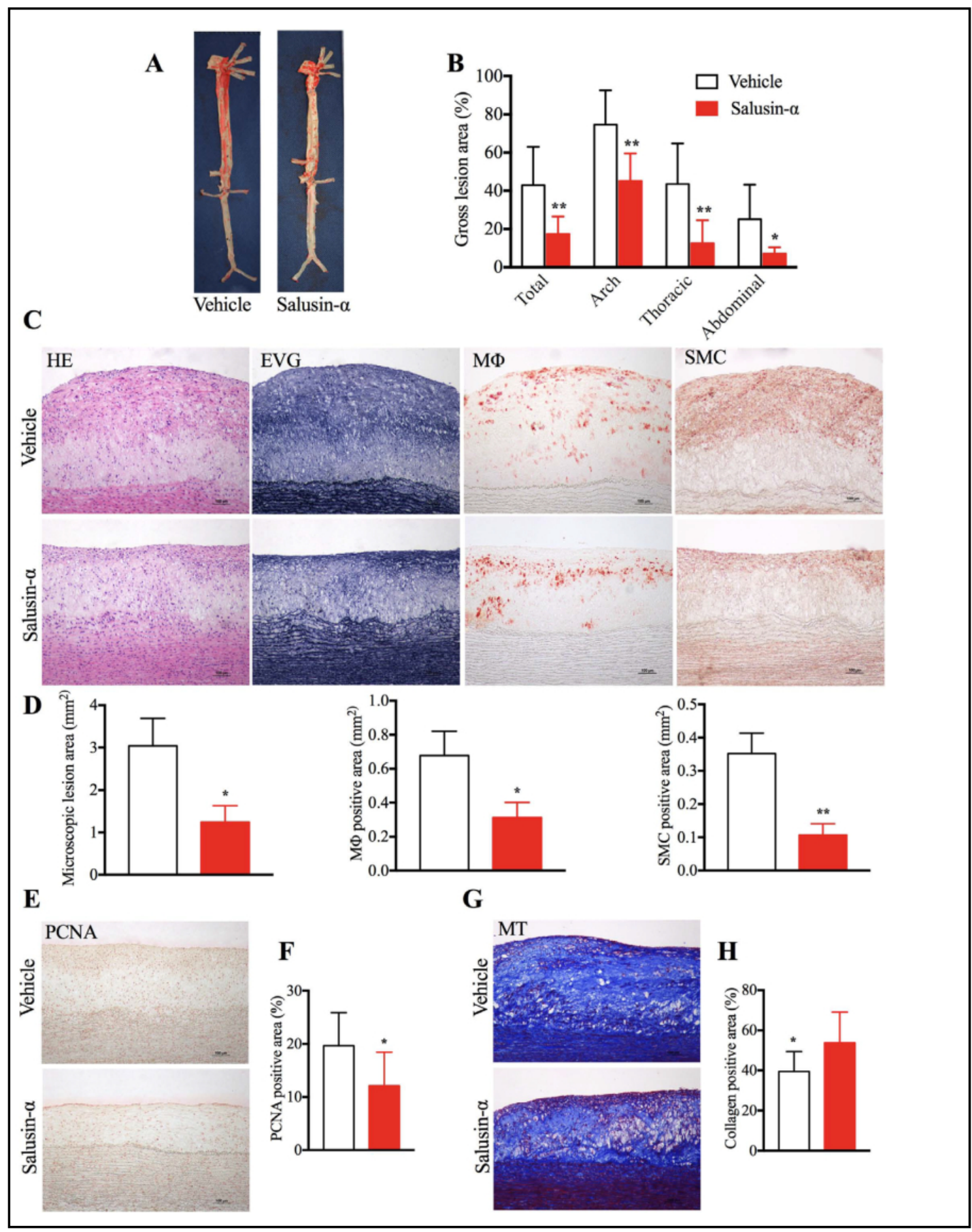

Fig. 2. Quantitation of aortic atherosclerosis. (A) Representative photographs of aortas stained with Sudan IV. (B) The lesion area (defined by the sudanophilic area) was quantified using an image analysis system. (C) Representative micrographs of aortic arch lesions. Serial paraffin sections of the aortic arch were stained with hematoxylin-eosin (HE) and elastica van Gieson (EVG) or immunohistochemically stained with monoclonal antibodies (mAbs) against either macrophages (MФ) or $\alpha$-SMA (smooth muscle actin) for smooth muscle cells (SMCs). (D) Quantitation of intimal lesions in EVG-stained sections and positively stained areas of M $\varphi$ and SMCs. Representative images (E) and quantitative data (F) of proliferating cell nuclear antigen (PCNA) staining in cross-sections of the aortic arch. Representative images (G) and quantitative data (H) stained with Masson's trichrome (MT). Data are expressed as the mean \pm standard deviation. $n=9$ for each group. ${ }^{*} \mathrm{P}<0.05$ and ${ }^{* *} \mathrm{P}<0.01$ versus the vehicle-treated group.

\section{KARGER}




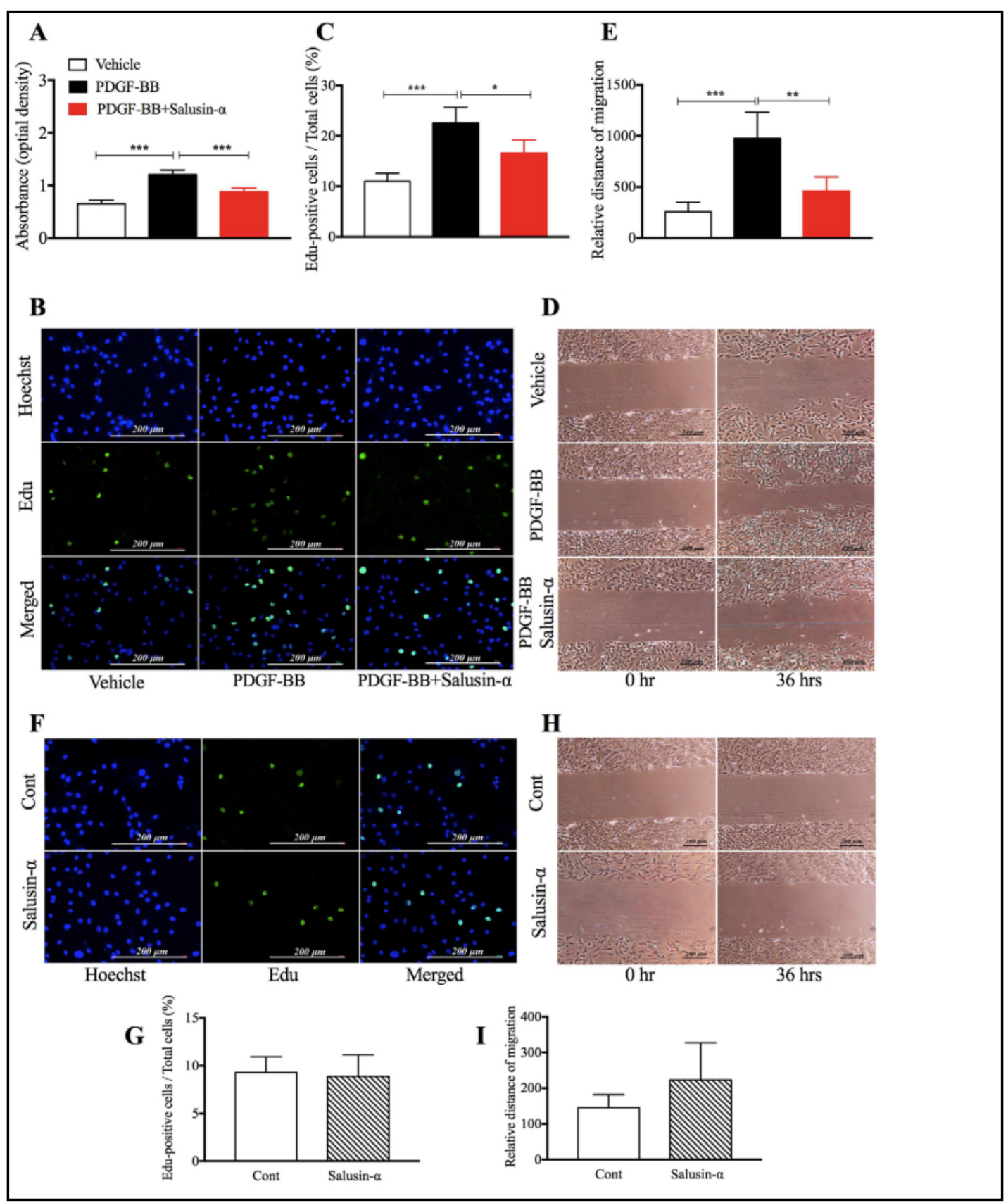

Fig. 3. Vascular smooth muscle cell (VSMC) proliferation and migration in vitro. (A) VSMCs were grown in a 96-well plate in the presence or absence of salusin- $\alpha(10 \mathrm{nM})$ with or without platelet-derived growth factor-BB (PDGF-BB) (10 ng/mL) for $36 \mathrm{~h}$. Representative images (B) and quantitative data (C) of the effect of salusin- $\alpha(10 \mathrm{nM})$ on the PDGF-BB-induced DNA synthesis of VSMCs determined with an 5-ethynyl-2'deoxyuridine (EdU) incorporation assay. The data are expressed as the ratio of EdU-positive cells to total cells. Blue fluorescence (Hoechst 33342) shows cell nuclei and green fluorescence (EdU) labels cells with DNA synthesis ( $\times 200)$. Representative images (D) and quantitative data (E) of the effect of salusin- $\alpha(10 \mathrm{nM})$ on the PDGF-BB-induced migration of VSMCs. VSMCs were scratched with a 1-mL pipette tip and cultured for $24 \mathrm{~h}$ with or without PDGF-BB (30 ng/mL). The dotted lines define the areas lacking cells. Representative images (F) and quantitative data $(G)$ of the effect of salusin- $\alpha(10 \mathrm{nM})$ on the DNA synthesis of VSMCs, determined with an EdU incorporation assay. Representative images (H) and quantitative data (I) of the effect of salusin- $\alpha(10 \mathrm{nM})$ on VSMC migration. Data are expressed as the mean \pm standard deviation. $\mathrm{n}=6$ for each group. ${ }^{*} \mathrm{P}<0.05$ and ${ }^{* *} \mathrm{P}<0.01$ versus the vehicle or $\mathrm{PDGF}-\mathrm{BB}-$ treated group. 


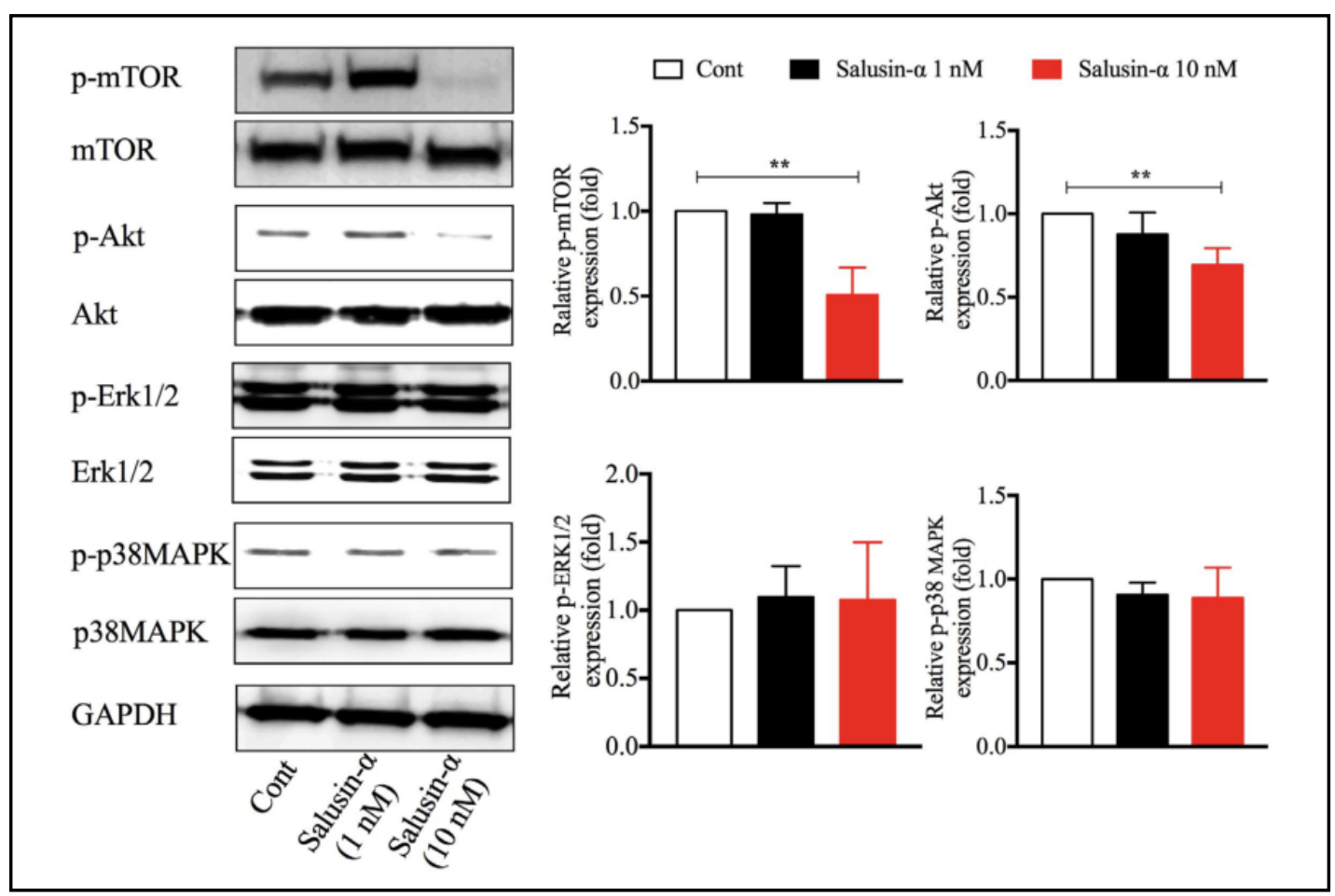

Fig. 4. PDGF-BB-stimulated phosphorylation of mTOR, Akt, ERK1/2, and p38 in VSMCs. Band densitometry is shown in the right panels. ERK1/2 indicates extracellular signal-regulated protein kinases $1 / 2 ; \mathrm{p}$-p38 MAPK, phospho-p38 mitogen-activated protein kinase; p-mTOR, phospho mammalian target of rapamycin. Data are mean \pm standard deviation of three independent experiments. ${ }^{* *} \mathrm{P}<0.01$ versus the PDGF-BBtreated group.

\section{VSMC proliferation and migration}

Physiological concentrations of salusin- $\alpha$ inhibited PDGF-BB-induced VSMC proliferation in a dose-dependent manner, with the maximal inhibition at $10 \mu \mathrm{M}$ (data not shown). PDGF-BB (10 ng/mL) increased VSMC proliferation 2-fold compared with vehicle treatment, whereas the increased proliferation induced by PDGF-BB was inhibited by $33 \%$ with $10 \mu \mathrm{M}$ salusin- $\alpha$ (Fig. 3A). We confirmed the suppressive effect of salusin- $\alpha$ on PDGFBB-induced VSMC proliferation with an EdU incorporation assay. EdU-positive cells in VSMC were increased $\sim 2$-fold over the control group by PDGF-BB (Fig. 3B and C). The increased DNA synthesis induced by PDGF-BB was inhibited by $34 \%$ with $10 \mathrm{nM}$ salusin- $\alpha$ (Fig. 3C). Furthermore, we examined the effect of salusin- $\alpha$ on VSMC migration in vitro. As shown in Fig. 3D and E, PDGF-BB increased VSMC migration 3.5-fold compared with vehicle treatment, but the effect was mitigated in the salusin- $\alpha$-treated group. Thus, these findings indicate that salusin- $\alpha$ may play a pivotal role in VSMC proliferation and migration, which are vital processes of atherosclerosis. These results suggest that salusin- $\alpha$ mitigated VSMC proliferation and migration in response to PDGF-BB. However, salusin- $\alpha$ exhibited no effect on proliferation (Fig. 3F and G) or migration (Fig. 3H and I) in cultured VSMCs without PDGFBB stimulation.

Signaling pathways activated during proliferation and migration in mitogen-treated VSMCS

To directly examine the cellular substrates that may explain the impaired proliferation and migration of VSMCs, we measured the phosphorylation levels of p38, Erk1/2, Akt, and mTOR after PDGF-BB stimulation with or without pretreatment with salusin- $\alpha$. As shown in Fig. 4, pAkt phosphorylation was inhibited by $26 \%$ by $10 \mathrm{nM}$ salusin- $\alpha$. Meanwhile, the 


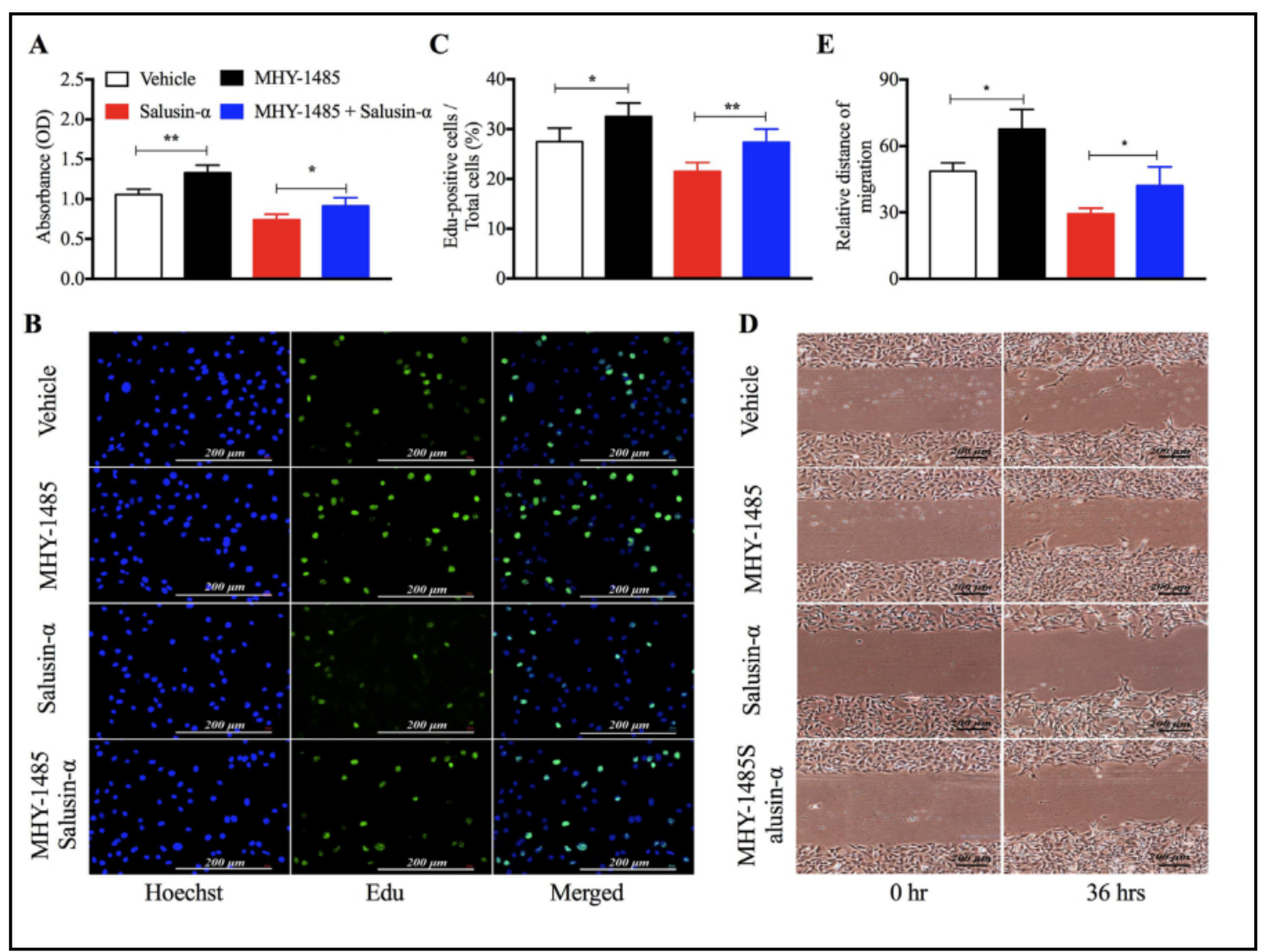

Fig. 5. MHY-1485 attenuates salusin- $\alpha$ mediated inhibition of PDGF-BB-induced VSMC proliferation and migration in vitro. VSMCs were treated with PDGF-BB $(10 \mathrm{ng} / \mathrm{mL})$ for $36 \mathrm{~h}$. (A) The CCK-8 assay was performed to assess the proliferation of VSMCs. Representative images (B) and quantitative data (C) from the EdU incorporation assay to assess DNA synthesis in VSMCs. Representative images (D) and quantitative data (E) of the wound-induced migration assay to assess the migration of VSMCs. Data are expressed as the mean \pm standard deviation. $\mathrm{n}=6$ for each group. ${ }^{*} \mathrm{P}<0.05$ and ${ }^{* *} \mathrm{P}<0.01$ versus the PDGF-BB- or salusin- $\alpha-$ treated group.

mTOR phosphorylation level was decreased by $49 \%$ after treatment with salusin- $\alpha(10 \mathrm{nM})$ compared with the PDGF-BB-treated group. However, salusin- $\alpha$ had no significant effect on the phosphorylation of Erk1/2 and p38. These results indicated that salusin- $\alpha$ inhibited PDGF-BB-induced proliferation and migration in VSMC by suppressing the Akt/mTOR signaling pathway. To further examine the role of Akt/mTOR signaling in PDGF-BB-induced VSMC proliferation and migration, MHY-1485 $(10 \mu \mathrm{M})$ was applied to activate the mTOR signaling pathway. As shown in Fig. 5A, MHY-1485 significantly attenuated the salusin- $\alpha-$ induced inhibition of VSMC proliferation. The result was confirmed by an EdU incorporation assay (Fig. 5B and C). Meanwhile, wound-healing assays showed that MHY-1485 significantly attenuated the salusin- $\alpha$-induced inhibition of VSMC migration (Fig. 5D and E).

\section{Matrix metalloproteinase expression in vivo and in vitro}

Because MMPs are involved in the progression of atherosclerosis, we examined MMP expression in the atherosclerotic lesions using immunohistochemical staining. The expression levels of MMP-2 and MMP-9 in the aortic plaque were markedly lower in the salusin- $\alpha$-treated group than in the vehicle-treated group (Fig. 6A and B). Furthermore, we investigated the effects of salusin- $\alpha$ on the mRNA expression levels of MMP-2 and MMP-9 in VSMCs. The mRNA expression levels (Fig. 6C) of MMP-2 and MMP-9 showed a marked increase in the PDGF-BB-treated group but were normalized in the salusin- $\alpha$-treated group compared with the vehicle-treated group.

\section{KARGER}


A
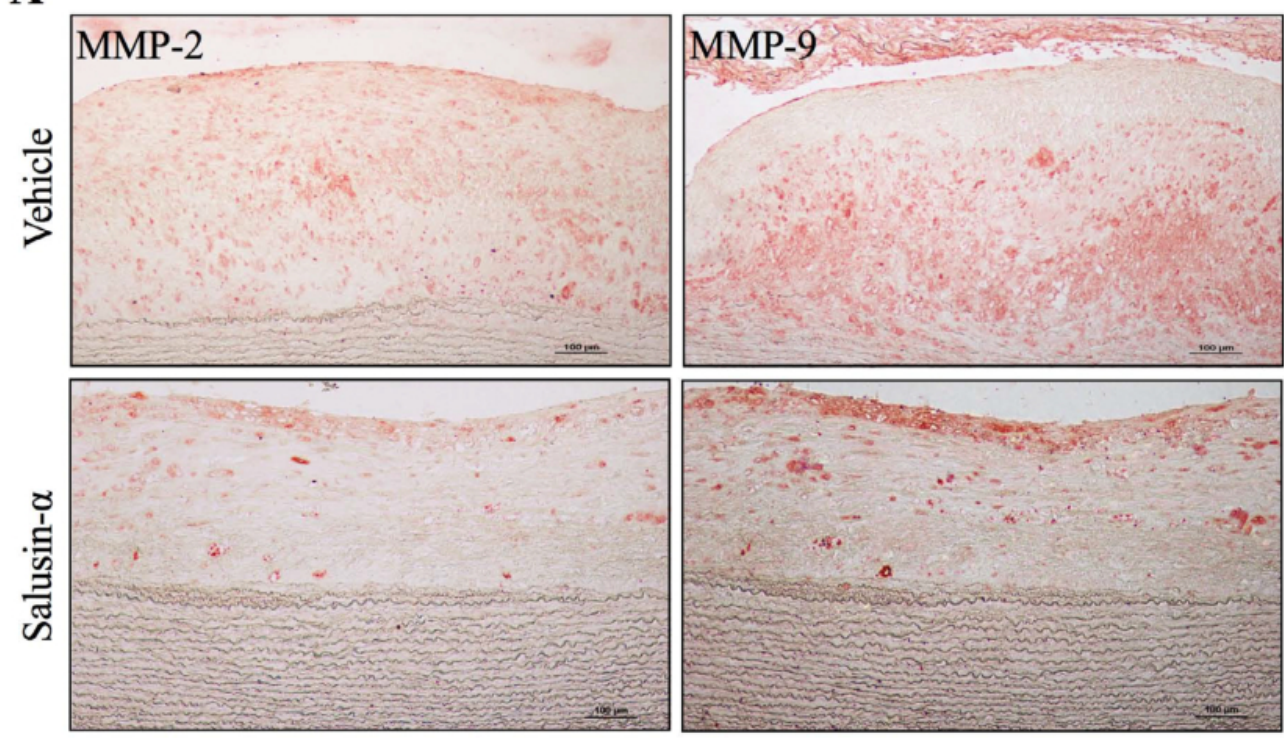

B
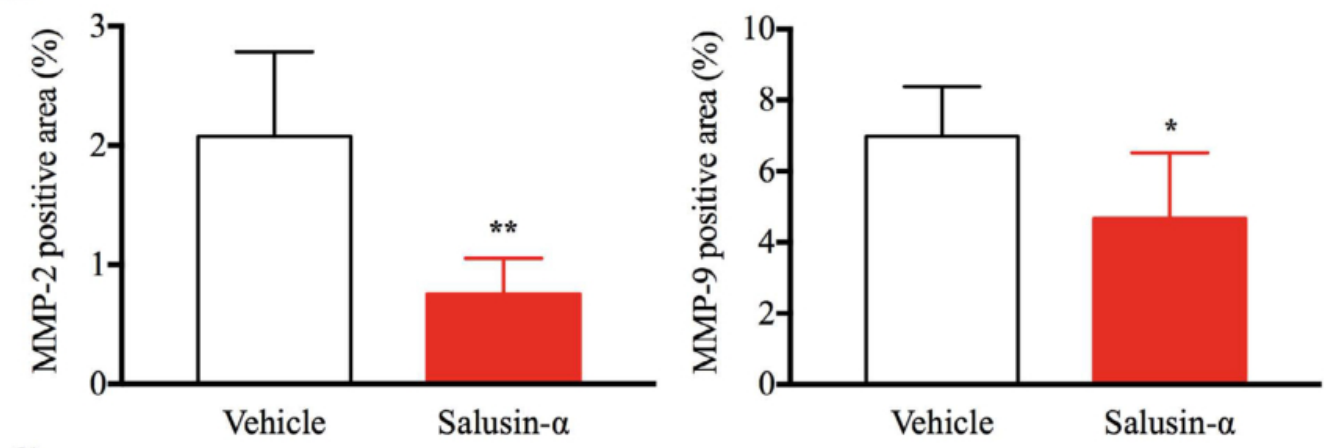

C
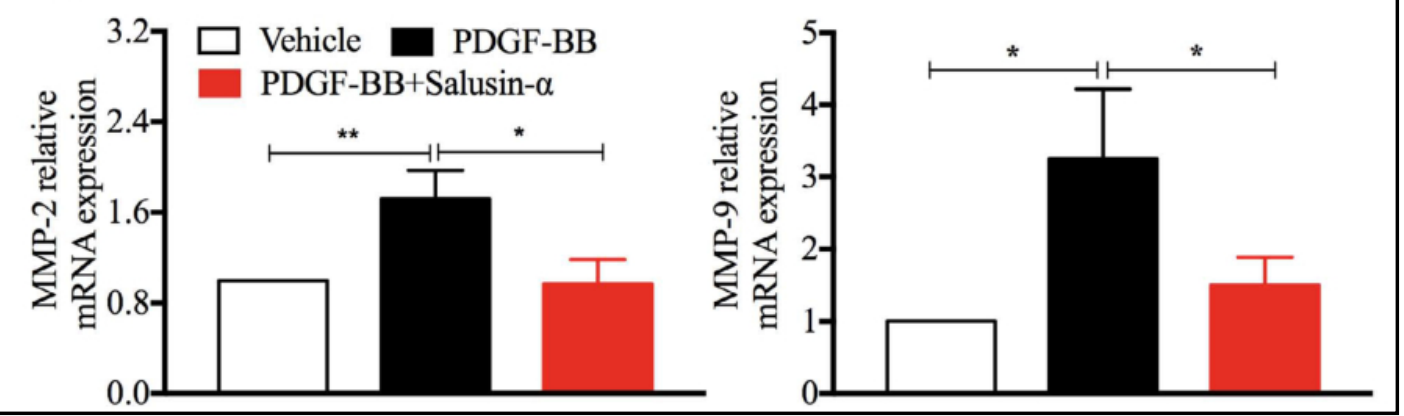

Fig. 6. Matrix metalloproteinase (MMP) expression in vivo and in vitro. Representative micrographs (A) of intimal lesions immunohistochemically stained with monoclonal antibodies against MMP-2 and MMP-9. (B) Quantitative analysis of positively stained areas of MMP-2 and MMP-9 with an image analysis system. Data are expressed as the mean \pm standard deviation. $\mathrm{n}=9$ for each group. ${ }^{*} P<0.05$ and ${ }^{* *} P<0.01$ versus the vehicle-treated group. (C) mRNA expression levels of MMP-2 and MMP-9 in vascular smooth muscle cells (VSMCs) with different treatments. Data are expressed as the mean \pm standard deviation. $\mathrm{n}=3$ for each group. ${ }^{*} \mathrm{P}<0.05$ and ${ }^{*} \mathrm{P}<0.01$ versus the vehicle or $\mathrm{PDGF}-\mathrm{BB}-$ treated group. 


\section{Cellular Physiology Cell Physiol Biochem 2018;50:1740-1753

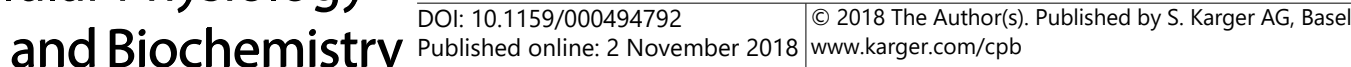 \\ Gao et al.: Salusin- $\alpha$ Inhibits Vascular Smooth Muscle Cell Proliferation}

\section{Discussion}

The significant finding of our present work is that chronic salusin- $\alpha$ infusion protects against cholesterol diet-induced atherosclerosis with a significant decrease in SMCs in atherosclerotic lesions. At the molecular level, salusin- $\alpha$ inhibits VSMC migration/ proliferation and MMP- 2 and MMP-9 expression and reduces Akt/mTOR signaling activation.

Nagashima et al. demonstrated that chronic salusin- $\alpha$ infusion blocks the progression of atherosclerotic lesions by suppressing serum TC levels in mice [15]. In our present study, no significant effects of salusin- $\alpha$ on plasma lipid levels were found in cholesterol-fed rabbits (Fig. 1); species differences might be one of the explanations for these apparently conflicting results, because rabbits have a unique lipoprotein metabolism profile (like humans but unlike rodents) and are sensitive to a cholesterol diet [24]. In rabbits, preprosalusin mRNA is widely expressed throughout most tissues but is especially abundant in the lung, spleen, testes, and hypothalamus, which is similar to human tissues. However, the human salusin- $\alpha$ Abs currently available do not cross-react with rabbit salusin and no rabbit salusin- $\alpha$ Abs are thus far available. Thus, it remains to be investigated whether rabbit salusin- $\alpha$ and salusin- $\beta$ colocalize or show distinct distributions.

In agreement with a previous report that salusin- $\alpha$ exerts protective effects in atherosclerosis through inhibition of macrophage-derived foam cells [16], we observed that chronic infusion of salusin- $\alpha$ substantially attenuated the aortic atherosclerotic lesions and inhibited both macrophage and SMC content in atherosclerotic lesions of rabbits (Fig. 2). VSMC proliferation and migration from the media into the intima are believed to be one of the main causes of the formation of atherosclerotic plaques and intimal hyperplasia [25]. Salusin- $\alpha$ decreases macrophage foam cell formation via acyl-coenzyme A:cholesterol acyltransferase- 1 regulation [16]. However, the role of salusin- $\alpha$ in another important cellular component of atherosclerotic lesions, VSMCs, remains to be clarified. In our present work, we observed decreased cell proliferative activity in the intima of arteriosclerotic arteries in the presence of salusin- $\alpha$ in vivo (Fig. 2) and found that salusin- $\alpha$ prevented mitogeninduced VSMC proliferation and migration in vitro (Fig. 3). The peptide that opposes salusin- $\alpha$, salusin- $\beta$, promotes the migration and proliferation of VSMCs and is involved in vascular remodeling $[19,20,26]$. VSMC proliferation in the development of atherosclerosis is most likely initiated and regulated by growth factors such as PDGF-BB. Akt-mTOR signaling has been accepted widely as an initiator of protein synthesis and cell growth. Akt plays a dominant role in VSMC growth and migration, whereas ERK, JNK, and p38 are comparably involved [27]. Akt and mTOR activation are important for regulating the phosphorylation of an array of substrates and mediating cellular processes such as cell migration, proliferation, survival, and the cell cycle [28-30]. Our results showed that salusin- $\alpha$ reduced the levels of phospho-Akt and phospho-mTOR proteins in VSMCs (Fig. 4), suggesting that the Akt/mTOR pathway may be involved in the effects of salusin- $\alpha$ on VSMC proliferation and migration. The subsequent wound "scratch" experiment confirmed that salusin- $\alpha$ can significantly inhibit PDGF-BB- or mTOR activator (MHY1485)-induced VSMC migration (Fig. 5). These results suggested that salusin- $\alpha$ might improve atherosclerosis by inhibiting VSMC migration and proliferation via regulation of the mTOR pathway. Although the salusin- $\alpha$ receptor has not yet been identified, we speculate that salusin- $\alpha$ may exert its physiological functions by binding to cell surface receptors [17, 31, 32]. Also unclear is whether salusin- $\alpha$ inhibits the migration and proliferation of SMCs via attenuation of the effects of salusin- $\beta$. Previous studies reported that pretreatment with excess salusin- $\alpha$ did not attenuate the salusin- $\beta-$ induced increase in intracellular calcium in VSMCs [10], suggesting that they do not compete for the same receptors. Because salusin- $\alpha$ and $-\beta$ have quite distinct sequences, we speculate that they may bind to different receptors, although this hypothesis has not been fully verified [33]. 


\section{Cellular Physiology Cell Physiol Biochem 2018;50:1740-1753

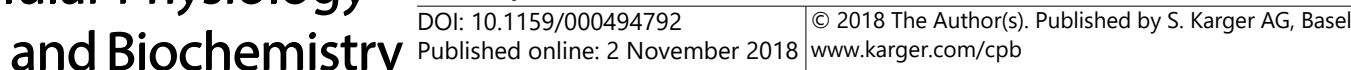 \\ Gao et al.: Salusin- $\alpha$ Inhibits Vascular Smooth Muscle Cell Proliferation}

A recent study reported that salusin- $\beta$, but not salusin- $\alpha$, promotes vascular inflammation in ApoE-deficient mice [34]. However, there was no significant difference between the two groups in the levels of IL-6, TNF- $\alpha$, or blood leukocytes (Fig. 1). Apart from inflammatory cytokines, macrophages and VSMCs in plaques may also secrete proteolytic enzymes, such as MMPs, which can digest the extracellular matrix, weaken the fibrous cap, and play an important role in the migration of cells in atherosclerotic lesions [35, 36]. VSMCs in culture often mimic the synthetic phenotype, typically expressing MMP-2 in the presence of an autocrine or PDGF loop [37]. Some peptides, such as salusin- $\beta$, promote the migration of VSMCs through upregulation of MMP-2 and MMP-9 [18, 38]. Here, we found that salusin- $\alpha$ treatment dramatically inhibited MMP-2 and MMP-9 expression in plaques (Fig. 6). More importantly, these primary findings in VSMCs were confirmed by our in vitro experiments (Fig. 6). Furthermore, the increased collagen content in the aortic plaques after salusin- $\alpha$ treatment may stem from the marked decrease in macrophages and MMPs in these lesions. These results suggest that salusin- $\alpha$ also might enhance plaque stability by targeting collagen accumulation, downregulating MMPs. Further studies will be needed to resolve this issue.

\section{Conclusion}

In this study, salusin- $\alpha$ was found to inhibit atherosclerotic lesions by suppressing VSMC proliferation and migration and to possibly also enhance plaque stability by regulating MMP expression and collagen production. The mechanisms underlying these beneficial effects on VSMCs may involve attenuation of the Akt/mTOR signaling pathways.

\section{Abbreviations}

Ab (antibody); CCK-8 (Cell Counting Kit-8); DMEM (Dulbecco's modified Eagle's medium); EdU (5-ethynyl-2'-deoxyuridine); ERK1/2 (extracellular signal-regulated protein kinases 1/2); FBS (fetal bovine serum); HDL-C (high-density lipoprotein-cholesterol); IL-6 (interleukin-6); MMP (matrix metalloproteinase); Mtor (mammalian target of rapamycin); PCNA (proliferating cell nuclear antigen); PDGF-BB (platelet-derived growth factor-BB); p38 (MAPK, p38 mitogen-activated protein kinase); SMC (smooth muscle cell); TC (total cholesterol); TG (triglycerides); TNF- $\alpha$ (tumor necrosis factor- $\alpha$ ); VSMC (vascular smooth muscle cell).

\section{Acknowledgements}

This study was supported by grants from the National Natural Science Foundation of China (81370379, 81070250, and 30900526 to EQ Liu and SH Zhao), the Natural Science Foundation of Shaanxi Province (2012KJXX-07 and 2014JQ4137 to SH Zhao and 2014FWPT07 to EQ Liu), and the Fundamental Research Fund for the Central Universities (to SH Zhao).

\section{Disclosure Statement}

The authors declare that they have no competing interests as defined by Cellular Physiology and Biochemistry or other interests that might be perceived to influence the results and discussion reported in this paper. 


\section{Cellular Physiology Cell Physiol Biochem 2018;50:1740-1753 \begin{tabular}{l|l|l}
\hline DOI: 10.1159/000494792 & ( 2018 The Author(s). Published by S. Karger AG, Basel
\end{tabular} and Biochemistry Published online: 2 November 2018 www.karger.com/cpb}

\section{References}

1 Lusis AJ: Atherosclerosis. Nature 2000;407:233-241.

2 van Diepen JA, Berbee JF, Havekes LM, Rensen PC: Interactions between inflammation and lipid metabolism: relevance for efficacy of anti-inflammatory drugs in the treatment of atherosclerosis. Atherosclerosis 2013;228:306-315.

- 3 Studentova H, Indrakova J, Petrova P, Kaminek M, Kalabova H, Sramek V, Adam T, Melichar B: Risk factors of atherosclerosis during systemic therapy targeting vascular endothelial growth factor. Oncol Lett 2016;11:939-944.

4 Kumar KG, Trevaskis JL, Lam DD, Sutton GM, Koza RA, Chouljenko VN, Kousoulas KG, Rogers PM, Kesterson RA, Thearle M, Ferrante AW, Jr., Mynatt RL, Burris TP, Dong JZ, Halem HA, Culler MD, Heisler LK, Stephens JM, Butler AA: Identification of adropin as a secreted factor linking dietary macronutrient intake with energy homeostasis and lipid metabolism. Cell Metab 2008;8:468-481.

-5 Kanome T, Watanabe T, Nishio K, Takahashi K, Hongo S, Miyazaki A: Angiotensin II upregulates acylCoA:cholesterol acyltransferase-1 via the angiotensin II Type 1 receptor in human monocyte-macrophages. Hypertens Res 2008;31:1801-1810.

6 Rocha NG, Templeton DL, Greiner JJ, Stauffer BL, DeSouza CA: Metabolic syndrome and endothelin-1 mediated vasoconstrictor tone in overweight/obese adults. Metabolism 2014;63:951-956.

7 Dimova R, Tankova T: The role of vaspin in the development of metabolic and glucose tolerance disorders and atherosclerosis. Biomed Res Int DOI: 10.1155/2015/823481.

-8 Watanabe T, Suguro T, Kanome T, Sakamoto Y, Kodate S, Hagiwara T, Hongo S, Hirano T, Adachi M, Miyazaki A: Human urotensin II accelerates foam cell formation in human monocyte-derived macrophages. Hypertension 2005;46:738-744.

Libby P, Ridker PM, Hansson GK: Progress and challenges in translating the biology of atherosclerosis. Nature 2011;473:317-325.

10 Shichiri M, Ishimaru S, Ota T, Nishikawa T, Isogai T, Hirata Y: Salusins: newly identified bioactive peptides with hemodynamic and mitogenic activities. Nat Med 2003;9:1166-1172.

11 Watanabe T, Sato K, Itoh F, Iso Y, Nagashima M, Hirano T, Shichiri M: The roles of salusins in atherosclerosis and related cardiovascular diseases. J Am Soc Hypertens 2011;5:359-365.

12 Kolakowska U, Olanski W, Wasilewska A: Salusins in hypertension and related cardiovascular diseases. Curr Drug Metab 2016;17:827-833.

13 Izumiyama H, Tanaka H, Egi K, Sunamori M, Hirata Y, Shichiri M: Synthetic salusins as cardiac depressors in rat. Hypertension 2005;45:419-425.

14 Suzuki N, Shichiri M, Tateno T, Sato K, Hirata Y: Distinct systemic distribution of salusin-alpha and salusinbeta in the rat. Peptides 2011;32:805-810.

15 Nagashima M, Watanabe T, Shiraishi Y, Morita R, Terasaki M, Arita S, Hongo S, Sato K, Shichiri M, Miyazaki A, Hirano T: Chronic infusion of salusin-alpha and -beta exerts opposite effects on atherosclerotic lesion development in apolipoprotein E-deficient mice. Atherosclerosis 2010;212:70-77.

-16 Watanabe T, Nishio K, Kanome T, Matsuyama TA, Koba S, Sakai T, Sato K, Hongo S, Nose K, Ota H, Kobayashi Y, Katagiri T, Shichiri M, Miyazaki A: Impact of salusin-alpha and -beta on human macrophage foam cell formation and coronary atherosclerosis. Circulation 2008;117:638-648.

17 Niepolski L, Grzegorzewska AE: Salusins and adropin: New peptides potentially involved in lipid metabolism and atherosclerosis. Adv Med Sci 2016;61:282-287.

-18 Sun HJ, Zhao MX, Ren XS, Liu TY, Chen Q, Li YH, Kang YM, Wang JJ, Zhu GQ: Salusin-beta promotes vascular smooth muscle cell migration and intimal hyperplasia after vascular injury via ROS/NFkappaB/MMP-9 pathway. Antioxid Redox Signal 2016;24:1045-1057.

19 Sun HJ, Liu TY, Zhang F, Xiong XQ Wang JJ, Chen Q Li YH, Kang YM, Zhou YB, Han Y, Gao XY, Zhu GQ: Salusinbeta contributes to vascular remodeling associated with hypertension via promoting vascular smooth muscle cell proliferation and vascular fibrosis. Biochim Biophys Acta 2015;1852:1709-1718.

20 Ren XS, Ling L, Zhou B, Han Y, Zhou YB, Chen Q Li YH, Kang YM, Zhu GQ: Silencing salusin-beta attenuates cardiovascular remodeling and hypertension in spontaneously hypertensive rats. Sci Rep DOI: 10.1038/ srep43259. 


\section{Cellular Physiology Cell Physiol Biochem 2018;50:1740-1753 \begin{tabular}{l|l|l|l} 
DOI: 10.1159/000494792 & 2018 The Author(s). Published by S. Karger AG, Basel
\end{tabular}

21 Zhao S, Li Y, Gao S, Wang X, Sun L, Cheng D, Bai L, Guan H, Wang R, Fan J, Liu E: Autocrine human urotensin II enhances macrophage-derived foam cell formation in transgenic rabbits. Biomed Res Int DOI: $10.1155 / 2015 / 8439592015$.

22 Wang Y, Cheng F, Chen Y, Li Y, Zhao S, Yu Q, Fan J, Liu E: High-dose rosiglitazone is pro-atherogenic in cholesterol-fed rabbits. Atherosclerosis 2012;222:292-294.

23 Wu H, Cheng XW, Hu L, Takeshita K, Hu C, Du Q Li X, Zhu E, Huang Z, Yisireyili M, Zhao G, Piao L, Inoue A, Jiang H, Lei Y, Zhang X, Liu S, Dai Q Kuzuya M, Shi GP, Murohara T: Cathepsin S activity controls injuryrelated vascular repair in mice via the TLR2-mediated p38MAPK and PI3K-Akt/p-HDAC6 signaling pathway. Arterioscler Thromb Vasc Biol 2016;36:1549-1557.

-24 Fan J, Kitajima S, Watanabe T, Xu J, Zhang J, Liu E, Chen YE: Rabbit models for the study of human atherosclerosis: from pathophysiological mechanisms to translational medicine. Pharmacol Ther 2015;146:104-119.

25 Mill C, George SJ: Wnt signalling in smooth muscle cells and its role in cardiovascular disorders. Cardiovasc Res 2012;95:233-240.

26 Sato K, Watanabe R, Itoh F, Shichiri M, Watanabe T: Salusins: potential use as a biomarker for atherosclerotic cardiovascular diseases. Int J Hypertens DOI: 10.1155/2013/965140.

27 Zhan Y, Kim S, Izumi Y, Izumiya Y, Nakao T, Miyazaki H, Iwao H: Role of JNK, p38, and ERK in plateletderived growth factor-induced vascular proliferation, migration, and gene expression. Arterioscler Thromb Vasc Biol 2003;23:795-801.

-28 Lawlor MA, Alessi DR: PKB/Akt: a key mediator of cell proliferation, survival and insulin responses? J Cell Sci 2001;114:2903-2910.

29 Somanath PR, Byzova TV: 14-3-3beta-Rac1-p21 activated kinase signaling regulates Akt1-mediated cytoskeletal organization, lamellipodia formation and fibronectin matrix assembly. J Cell Physiol 2009;218:394-404.

-30 Althoff TF, Albarran Juarez J, Troidl K, Tang C, Wang S, Wirth A, Takefuji M, Wettschureck N, Offermanns $\mathrm{S}$ : Procontractile G protein-mediated signaling pathways antagonistically regulate smooth muscle differentiation in vascular remodeling. J Exp Med 2012;209:2277-2290.

-31 Esfahani M, Saidijam M, Goodarzi MT, Movahedian A, Najafi R: Salusin-alpha attenuates inflammatory responses in vascular endothelial cells. Biochemistry (Mosc) 2017;82:1314-1323.

-32 Bader M, Alenina N, Andrade-Navarro MA, Santos RA: MAS and its related G protein-coupled receptors, Mrgprs. Pharmacol Rev 2014;66:1080-1105.

-33 Tanyeli A, Eraslan E, Polat E, Bal T: Protective effect of salusin-alpha and salusin-beta against ethanolinduced gastric ulcer in rats. J Basic Clin Physiol Pharmacol 2017;28:623-630.

-34 Zhou CH, Liu L, Liu L, Zhang MX, Guo H, Pan J, Yin XX, Ma TF, Wu YQ: Salusin-beta not salusin-alpha promotes vascular inflammation in ApoE-deficient mice via the I-kappaBalpha/NF-kappaB pathway. PLoS One DOI: 10.1371/journal.pone.0091468.

-35 Visse R, Nagase H: Matrix metalloproteinases and tissue inhibitors of metalloproteinases: structure, function, and biochemistry. Circ Res 2003;92:827-839.

-36 Liang J, Liu E, Yu Y, Kitajima S, Koike T, Jin Y, Morimoto M, Hatakeyama K, Asada Y, Watanabe T, Sasaguri Y, Watanabe S, Fan J: Macrophage metalloelastase accelerates the progression of atherosclerosis in transgenic rabbits. Circulation 2006;113:1993-2001.

37 Gough PJ, Gomez IG, Wille PT, Raines EW: Macrophage expression of active MMP-9 induces acute plaque disruption in apoE-deficient mice. J Clin Invest 2006;116:59-69.

-38 Park HJ, Kim MK, Kim Y, Bae SS, Kim HJ, Bae SK, Bae MK: Gastrin-releasing peptide promotes the migration of vascular smooth muscle cells through upregulation of matrix metalloproteinase-2 and -9. BMB Rep 2017;50:628-633. 